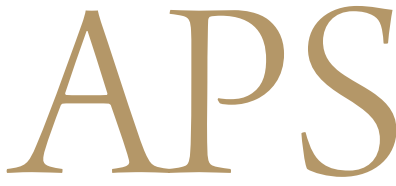

Archives of Plastic Surgery

\title{
Use of the Anterolateral Thigh and Vertical Rectus Abdominis Musculocutaneous Flaps as Utility Flaps in Reconstructing Large Groin Defects
}

\author{
Edwin Jonathan Aslim ${ }^{1}$, Mohamed Zulfikar Rasheed ${ }^{2}$, Fangbo Lin ${ }^{3}$, Yee-Siang Ong ${ }^{2}$, \\ Bien-Keem Tan ${ }^{2}$ \\ ${ }^{1}$ Department of Urology, Singapore General Hospital, Singapore; ${ }^{2}$ Department of Plastic, Reconstructive and Aesthetic Surgery, Singapore \\ General Hospital, Singapore; ${ }^{3}$ University of Auckland School of Medicine, Auckland, New Zealand
}

Background Groin dissections result in large wounds with exposed femoral vessels requiring soft tissue coverage, and the reconstructive options are diverse. In this study we reviewed our experience with the use of the pedicled anterolateral thigh and vertical rectus abdominis musculocutaneous flaps in the reconstruction of large groin wounds.

Methods Groin reconstructions performed over a period of 10 years were evaluated, with a mean follow up of two years. We included all cases with large or complex (involving perineum) defects, which were reconstructed with the pedicled anterolateral thigh musculocutaneous or the vertical rectus abdominis musculocutaneous (VRAM) flaps. Smaller wounds which were covered with skin grafts, locally based flaps and pedicled muscle flaps were excluded.

Results Twenty-three reconstructions were performed for large or complex groin defects, utilising the anterolateral thigh $(n=10)$ and the vertical rectus abdominis $(n=13)$ pedicled musculocutaneous flaps. Femoral vein reconstruction with a prosthetic graft was required in one patient, and a combination flap (VRAM and gracilis muscle flap) was performed in another. Satisfactory coverage was achieved in all cases without major complications. No free flaps were used in our series.

Conclusions The anterolateral thigh and vertical rectus abdominis pedicled musculocutaneous flaps yielded consistent results with little morbidity in the reconstruction of large and complex groin defects. A combination of flaps can be used in cases requiring extensive cover.

Keywords Groins / Surgical flap / Pedicled flap / Musculocutaneous flap
Correspondence: Bien-Keem Tan Department of Plastic, Reconstructive and Aesthetic Surgery, Singapore General Hospital, Outram Road, Singapore 169608, Singapore

Tel: $+65-6321-4686$

Fax: +65-6225-9340

E-mail: bienkeem@gmail.com

Received: 4 May 2014• Revised: 27 Aug 2014 • Accepted: 27 Aug 2014

pISSN: 2234-6163 • elSSN: 2234-6171 • http://dx.doi.org/10.5999/aps.2014.41.5.556 • Arch Plast Surg 2014;41:556-561

No potential conflict of interest relevant to this article was reported.

\section{INTRODUCTION}

Groin dissections performed for metastatic disease to the inguinal nodes from malignancies of the urogenitalia or lower limbs can leave wounds that are difficult to close by primary intention. The extent of the wound defect is dependent on the volume of the nodal metastases, with bulky disease requiring extensive resection. The femoral vessels are exposed following inguinal lymphadenectomy, leaving the area liable to complications such as femoral blowouts. To prevent this, a good soft tissue cover is essential.

Groin dissections are performed either as part of curative sur- 
gery, or in palliative resection where the nodes are fungating or ulcerating. In patients with bulky nodal disease, prognosis is poor even in the setting of curative surgery. For example, the 5-year survival rate for stage 3 squamous cell carcinoma of the penis is $30 \%$ [1]. Thus, the role of the reconstructive surgeon is to choose the most suitable coverage with the least impairment to quality of life, with a good balance between functional outcome and minimal donor morbidity. Furthermore, the wound needs to heal fast to reduce the delay in adjuvant therapy. Various options are available for the reconstruction of small defects, including locally-based fasciocutaneous or regional muscle flaps [2-4]. The use of skin grafts is not suitable when the femoral vessels are exposed. Large defects require a greater amount of soft tissue cover, which is available with the use of musculocutaneous flaps. We review our experience in the coverage of large and complex groin defects utilising the anterolateral thigh and vertical rectus abdominis pedicled musculocutaneous flaps as our main workhorses, and advocate their routine use.

\section{METHODS}

All groin reconstruction cases performed by our senior author (B.K.T.) over a 10 year period from January 2002 to December
2012, were evaluated. We excluded cases with small groin defects which were covered with skin grafts, locally based cutaneous flaps and pedicled muscle flaps. Twenty-three cases with groin dissections resulting in large or complex groin defects were included in our series (Table 1), and were all reconstructed with either the pedicled anterolateral thigh musculocutaneous flap or vertical rectus abdominis pedicled musculocutaneous flap. The underlying pathological conditions for this group included: squamous cell carcinoma of the penis $(n=10)$, vulva $(n=6)$, scrotum $(n=1)$, groin $(n=1)$, lower limb $(n=2)$; transitional cell carcinoma of bladder $(n=1)$; invasive extramammary Paget's disease $(n=1)$ and lower limb melanoma $(n=1)$. No free flap was used in our series.

All groin defects in the 23 cases were greater than $15 \mathrm{~cm} \times 10$ $\mathrm{cm}$, and categorised as large $(\mathrm{n}=20)$ or complex (involving perineum or requiring vascular reconstruction, $n=3$ ). Of these, 10 cases $(43.5 \%)$ were reconstructed with the anterolateral thigh flap, and the remaining 13 (56.5\%) were resurfaced with the vertical rectus abdominis flap: contralateral $(n=6)$ and ipsilateral $(n=7)$. A few factors were considered when choosing between the two. In patients with previous lower abdominal surgery and in obese patients, where harvesting the rectus abdominis flap would be challenging, the anterolateral thigh flap was

Table 1. Patient characteristics and methods of wound coverage

\begin{tabular}{|c|c|c|c|c|c|c|}
\hline $\begin{array}{l}\text { Patient } \\
\text { no. }\end{array}$ & Age $(y r) / S e x$ & Underlying diagnosis & Wound defect (cm) & Coverage & Donor closure & Complications \\
\hline 1 & 73/Male & SCC penis & Large & ALT MC & Primary & - \\
\hline 2 & 66/Male & SCC penis, groin recurrence & Large & ALT MC & SSG & - \\
\hline 3 & 70/Female & Melanoma heel & Large & ALT MC & SSG & - \\
\hline 4 & 63/Female & Paget's perineum invasive & Large & ALT MC & SSG & - \\
\hline 5 & 57/Female & SCC vulva & Large & ALT MC & SSG & Deep vein thrombosis \\
\hline 6 & 65/Male & SCC scrotum & Large & ALT MC & SSG & - \\
\hline 7 & 48/Male & SCC penis & Large & ALT MC & SSG & - \\
\hline 8 & 67/Male & TCC bladder & Large & ALT MC & SSG & - \\
\hline 9 & 50/Male & SCC penis & Large & ALT MC & SSG & - \\
\hline 10 & 78/Male & SCC penis & Large & ALT MC & SSG & - \\
\hline 11 & 78/Male & SCC penis, groin recurrence & Large & VRAM MC -C & Bridging mesh & - \\
\hline 12 & 65/Male & SCC penis & Large & VRAM MC -C & Bridging mesh & - \\
\hline 13 & 67/Female & SCC vulva & Large & VRAM MC -C & Bridging mesh & Flap cellulitis \\
\hline 14 & 77/Female & SCC vulva & Complex & VRAM MC -C & Bridging mesh & - \\
\hline 15 & 74/Male & SCC groin adherent to femoral vein & Complex & VRAM MC -C & Bridging mesh & - \\
\hline 16 & 64/Female & SCC vulva & Complex & VRAM MC -C & Bridging mesh & - \\
\hline 17 & 66/Male & SCC penis & Large & VRAM MC -i & Bridging mesh & - \\
\hline 18 & 51/Male & SCC penis & Large & VRAM MC -i & Primary & Donor wound edge necrosis \\
\hline 19 & 53/Male & SCC penis & Large & VRAM MC -i & Primary & - \\
\hline 20 & 47/Male & SCC ankle & Large & VRAM MC -i & Bridging mesh & - \\
\hline 21 & 59/Female & SCC vulva & Large & VRAM MC -i & Bridging mesh & - \\
\hline 22 & 71/Female & SCC vulva & Large & VRAM MC -i & Primary & - \\
\hline 23 & 49/Male & SCC foot & Large & VRAM MC -i & Primary & - \\
\hline
\end{tabular}




\section{Fig. 1. ALT pedicled flap}

(A) Large groin defect post resection of a penile squamous cell carcinoma with bulky inguinal lymphadenopathy. (B) Pedicled anterolateral thigh (ALT) flap was raised and inset over the groin wound. (C) Postoperative results at two months.
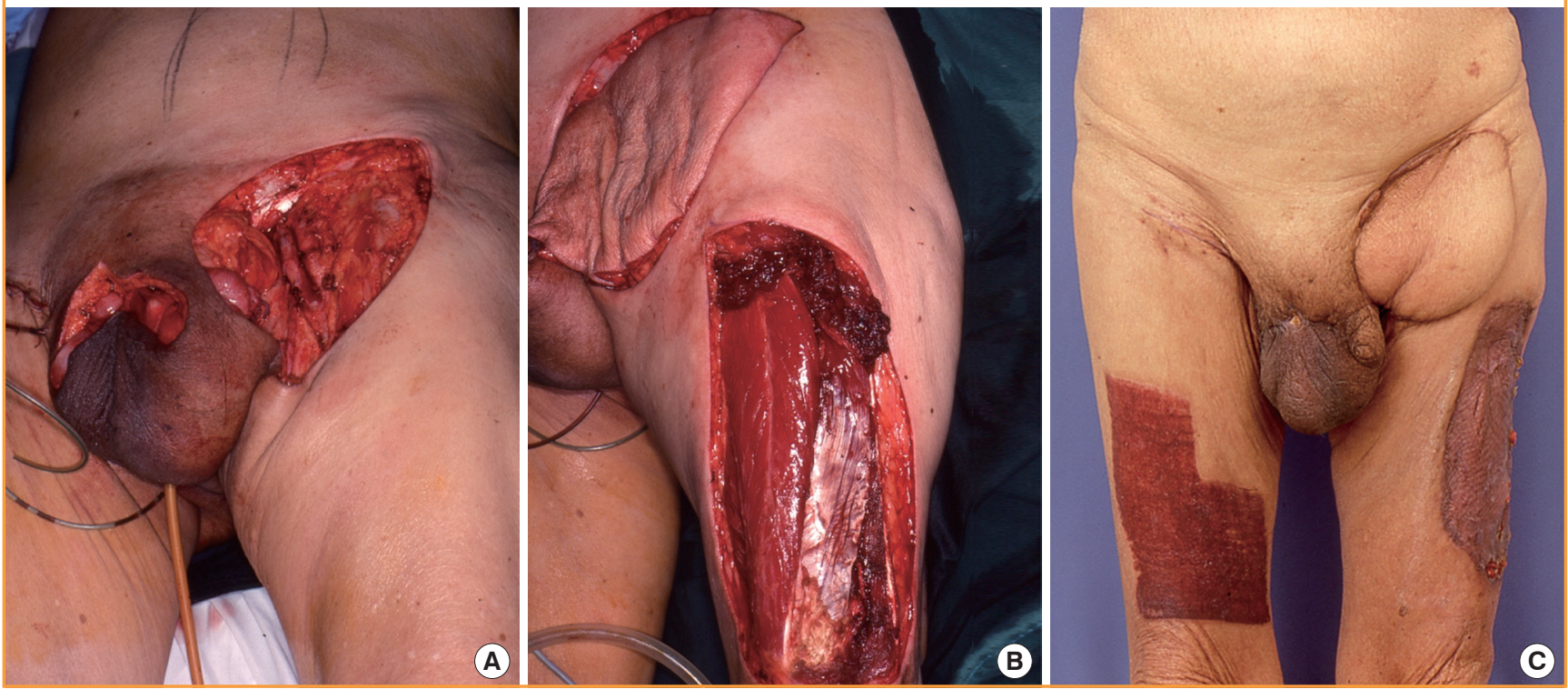

Fig. 2. Ipsilateral VRAM pedicled flap

(A) Post resection defects for penile squamous cell carcinoma with fungating lymphadenopathy. (B) An ipsilateral vertical rectus abdominis (VRAM) flap was raised and inset over the groin wound. (C) Postoperative results at four months.

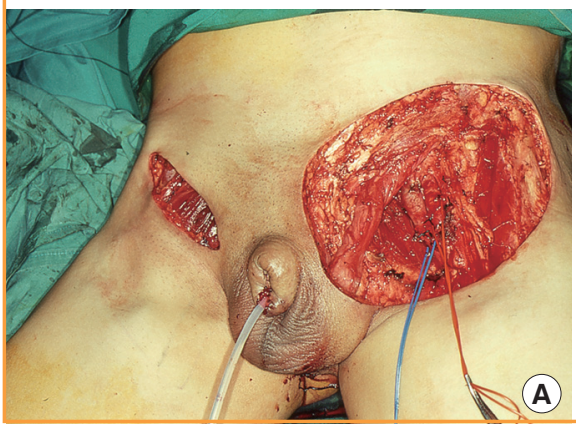

(A)

used (Fig. 1). The vertical rectus abdominis flap was used for complex defects (Fig. 2) and for patients in whom deep vein thrombosis was of particular concern.

All flaps were raised in the standard fashion. The skin paddle for the anterolateral thigh flap was designed with the aid of handheld Doppler ultrasound, and centred over the loudest audible signal. The anterolateral thigh flap donor sites were skin grafted in all but one patient. Abdominal wall fascial defects resulting from raising the vertical rectus abdominis flap were closed by primary fascial repair $(n=4)$ or by placing bridging meshes $(n=9)$. In cases with scrotal defects, primary closure of the scrotum was achieved using the scrotal advancement flap technique [5]. Two illustrative cases in the group with the vertical rectus abdominis flap are described as follows:

The patient of case 14 (Fig. 3) with a squamous cell carcinoma of the vulva had a complex groin defect extending to the perineum after tumour resection. The groin defect was covered with a contralateral pedicled vertical rectus abdominis flap, in combi- 


\section{Fig. 3. Contralateral VRAM pedicled flap with gracilis muscle flap}

(A) Complex groin defects extending to the perineum after clearance of a vulvar squamous cell carcinoma with bulky lymphadenopathy. A right subcostal scar from previous surgery was noted. $(B, C)$ A contralateral vertical rectus abdominis (VRAM) flap raised and inset over the groin defect. (D, E) The vulva/perineum defect was reconstructed with a pedicled gracilis muscle flap and skin grafted. (F) Postoperative results at four months.
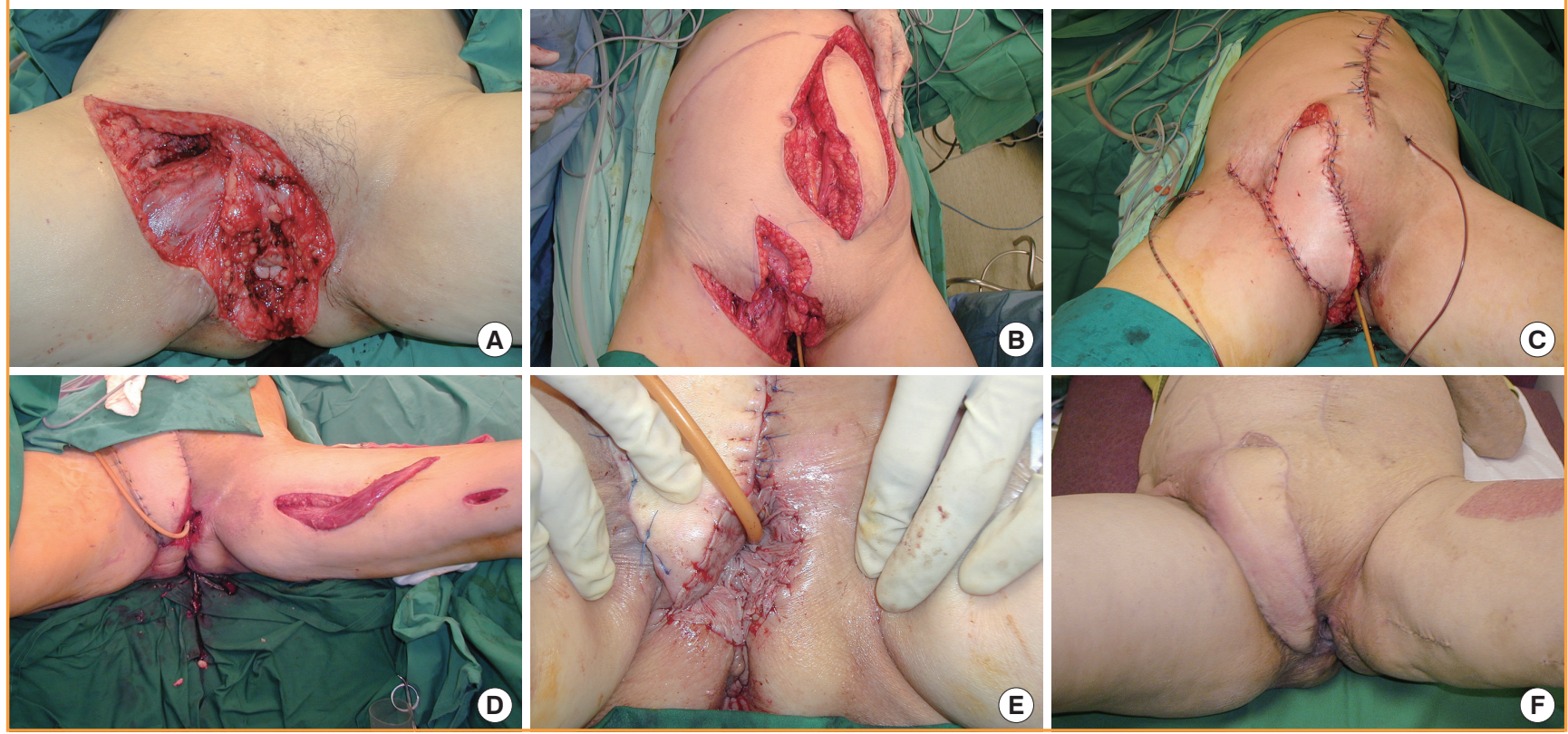

Fig. 4. Contralateral VRAM pedicled flap over vascular prosthesis

(A) Complex groin defect post en-bloc resection of a fungating groin squamous cell carcinoma adherent to the femoral vein. The femoral vein was reconstructed using a polytetrafluroethylene (PTFE) vascular graft. (B) A contralateral vertical rectus abdominis (VRAM) flap was inset in a manner that afforded total muscle wrap of the PTFE graft. (C) Postoperative results at two months.
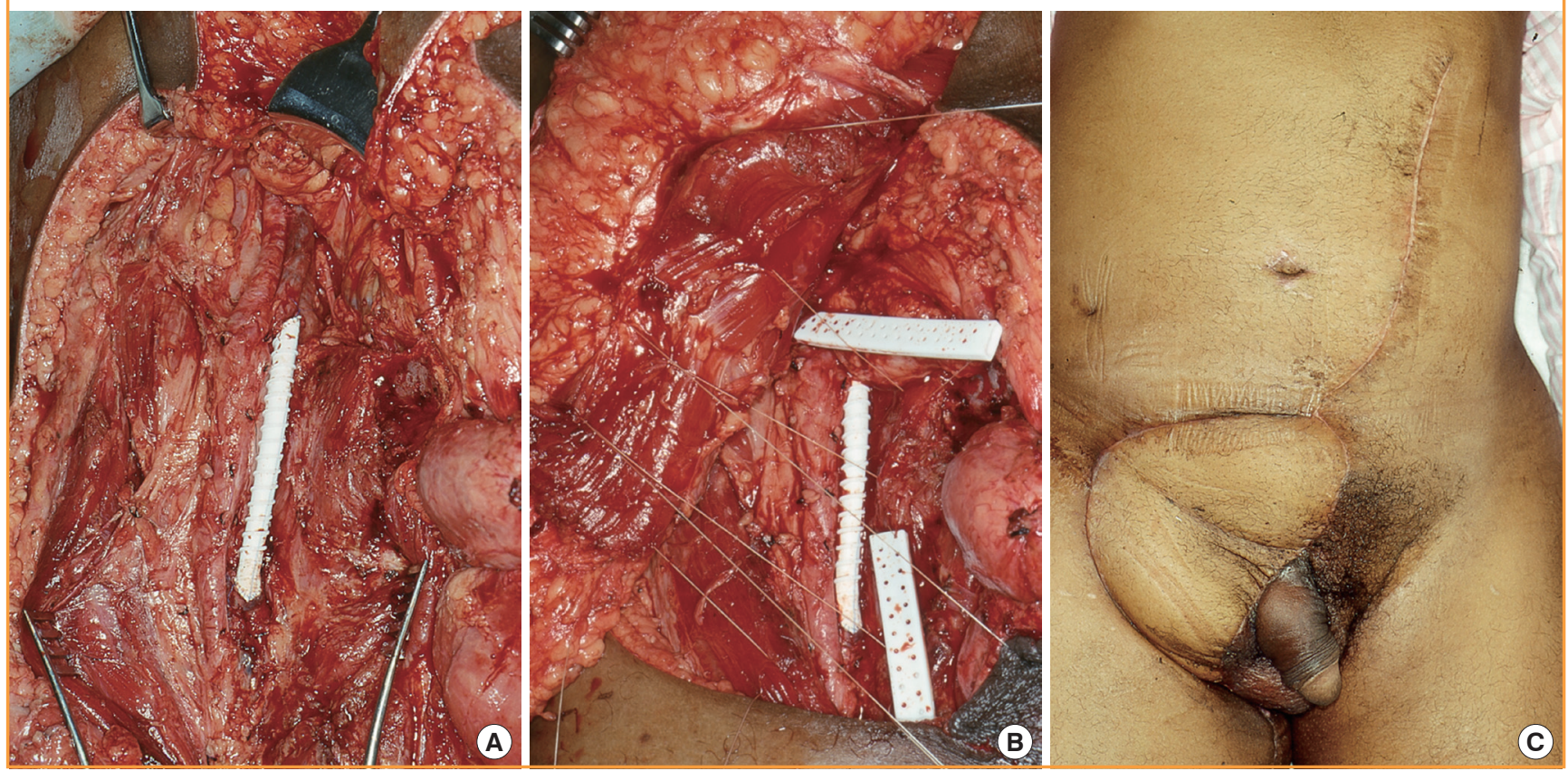

nation with a locally based transposition flap over the superior aspect of the wound. A second stage surgery was performed to reconstruct the vulva using a pedicled gracilis muscle, which was skin grafted. The patient recovered well.
In case 15 (Fig. 4), the patient had en-bloc resection of a squamous cell carcinoma of the groin which was adherent to the femoral vein. The femoral vein was reconstructed with a polytetrafluroethylene (PTFE) vascular graft. A contralateral vertical 
rectus abdominis flap was used to cover the groin defect, and inset with the muscle belly completely wrapping the prosthesis. The vascular conduit remained patent postoperatively.

\section{RESULTS}

No long-term or major complications such as flap loss or significant donor morbidities were encountered during an average follow-up period of two years; in particular there was no flap compromise in those patients receiving postoperative radiotherapy. Functional and cosmetic results were good.

In the group with the anterolateral thigh flap, the patient of case 5 had thrombosis in the femoral vein ipsilateral to the flap. The patient presented a few days postoperatively with a swollen leg and congested flap, with the Duplex scan confirming an acute femoral vein thrombosis. Anticoagulation therapy was instituted with low molecular weight heparin and the flap was closely monitored. The venous thrombosis resolved after a few weeks and the flap survived.

Two minor complications were observed in the group with the vertical rectus abdominis flap. One patient had wound edge necrosis of the donor site, which was debrided and later skin grafted successfully. Another patient with flap cellulitis was treated with antibiotics, and the infection resolved uneventfully.

\section{DISCUSSION}

In the reconstruction of large and complex groin defects, the use of musculocutaneous flaps is advantageous as they provide a large amount of soft tissue and tolerate radiotherapy well. By providing durable coverage they prevent scar contractures, which tend to form in flexural regions such as the groin. While primary closure of large groin defects is possible using local advancement flaps [6], the risk of wound breakdown is high as branches of the external iliac and femoral arteries supplying the region are divided during groin dissection, rendering the surrounding tissue relatively ischaemic. These branches include the superficial circumflex iliac artery, the superficial epigastric artery, and the superficial and deep external pudendal arteries.

We raised the musculocutaneous flaps as pedicled flaps in this series. The use of free flaps is an option in cases with extensive defects, but the main drawback to this method is the long operating time. Various options exist for the choice of pedicled musculocutaneous flaps, including the sartorious, rectus femoris, tensor fascia lata, anterolateral thigh and rectus abdominis flaps [4,7-10]. Although the use of the sartorious flap is associated with minimal donor morbidiy, it is too thin and has a limited arc of rotation owing to its segmental blood supply. The bulkier rec- tus femoris muscle provides better coverage but can cause weakness in knee extension $[4,8]$. A reliable flap to use is the tensor fascia lata flap which is based on the ascending branch of the lateral circumflex femoral artery. Because of its proximity to the groin, it is a workhorse flap for the region and has been used successfully for large defects $[11,12]$. It is associated with minimal functional morbidity, and the donor site can be skin grafted. We did not use the tensor fascia lata flap in this series. The anterolateral thigh and vertical rectus abdominis musculocutaneous flaps are versatile and reliable, and they are our first choice for groin reconstruction.

The anterolateral thigh flap comprises a large skin paddle with a cuff of vastus lateralis muscle. The advantages of this flap include its large skin paddle and its relatively problem-free donor site. For groin wounds extending medially, the reach of the flap can be extended by tunnelling it under the rectus femoris for a more direct route. The disadvantages of the anterolateral thigh flap include its downstream location to the groin defect, where its venous circulation may be compromised in cases of femoral vein compression by tumour. Since its blood supply arises distal to the groin, it could potentially be damaged or be prone to thrombosis if there is excessive manipulation of the femoral vessels during tumour clearance. Thrombosis of the femoral vein itself, as was the case in one patient (case 5), could also compromise flap survival. Hence we advise against using the anterolateral thigh flap in patients with bulky lymphadenopathy or in those predisposed to deep vein thrombosis. Primary closure of the donor site, being near the groin wound, results in a mutually opposing tension across adjacent defects. This may compromise flap survival or worse, thigh circulation. In our series we were able to close the donor site primarily only in one obese patient because of skin laxity. We used the handheld Doppler for our skin paddle design preoperatively. Significant variability in the perforator anatomy of the anterolateral thigh flap has been reported, and there is a recent move towards performing preoperative computed tomography angiography in aid of perforator selection [13].

The vertical rectus abdominis flap is used for the coverage of complex groin defects, or as an alternative to the anterolateral thigh flap in situations where femoral vein thrombosis was of concern. The advantages of this flap include: its upstream location in relation to the groin, the option of using a contralateral flap, and the ability to close the donor site primarily. Its blood supply originates proximal to the groin, and is at lower risk of damage during tumour clearance compared to downstream flaps. If the inferior epigastric vessels are divided during radical groin dissection, a contralateral flap can still be used. In case 15 where the patient required prosthetic grafting of the femoral vein, the choice for the contralateral flap was made so as to avoid 
flap compromise secondary to infection or thrombosis with proximal clot propagation. The disadvantages of this flap relate to donor morbidity, which include: abdominal bulge, hernia at the pivot point, and mesh infection. None of these complications occurred in our series. We routinely used bridging meshes to repair wide fascial defects to achieve tension-free repair.

Perforator-based flaps are increasingly popular in the reconstruction of the perineum $[14,15]$. Both the perforator-based anterolateral thigh flap and the deep inferior epigastric perforator flap are suitable options for groin reconstruction with less muscle damage. However, we still prefer musculocutaneous flaps in contaminated wounds, or if a vascular prosthesis is used. Unlike free flaps, a cuff of muscle must still be cut for the flap to move.

For complex wounds necessitating a large amount of soft tissue cover, a combination of flaps can be used. The only case in our series (case 14) utilised a contralateral vertical rectus abdominis flap in combination with a pedicled gracilis muscle flap to reconstruct the groin and vulvar/perineal wounds. Other forms of combination flaps have been described in the literature, including the chimeric flap such as the tensor fascia lata-sartorious and the tensor fascia lata-rectus femoris musculocutaneous flaps $[7,16]$.

\section{REFERENCES}

1. Horenblas S, van Tinteren H. Squamous cell carcinoma of the penis. IV. Prognostic factors of survival: analysis of tumor, nodes and metastasis classification system. J Urol 1994;151:1239-43.

2. Maser B, Vedder N, Rodriguez D, et al. Sartorius myoplasty for infected vascular grafts in the groin. Safe, durable, and effective. Arch Surg 1997;132:522-5.

3. Chen SH, Hentz VR, Wei FC, et al. Short gracilis myocutaneous flaps for vulvoperineal and inguinal reconstruction. Plast Reconstr Surg 1995;95:372-7.

4. Alkon JD, Smith A, Losee JE, et al. Management of complex groin wounds: preferred use of the rectus femoris muscle flap. Plast Reconstr Surg 2005;115:776-83.

5. Por YC, Tan BK, Hong SW, et al. Use of the scrotal remnant as a tissue-expanding musculocutaneous flap for scrotal reconstruction in Paget's disease. Ann Plast Surg 2003;51:
155-60.

6. Tabatabaei S, McDougal WS. Primary skin closure of large groin defects after inguinal lymphadenectomy for penile cancer using an abdominal cutaneous advancement flap. J Urol 2003;169:118-20.

7. Koshima I, Soeda S, Ohno A. Combined tensor fasciae latae musculocutaneous flap and sartorius musculocutaneous flap for the repair of wide defects of the lower leg. Plast Reconstr Surg 1990;86:1198-201.

8. Caulfield WH, Curtsinger L, Powell G, et al. Donor leg morbidity after pedicled rectus femoris muscle flap transfer for abdominal wall and pelvic reconstruction. Ann Plast Surg 1994;32:377-82.

9. Evriviades D, Raurell A, Perks AG. Pedicled anterolateral thigh flap for reconstruction after radical groin dissection. Urology 2007;70:996-9.

10. Creagh TA, Dixon L, Frizelle FA. Reconstruction with vertical rectus abdominus myocutaneous flap in advanced pelvic malignancy. J Plast Reconstr Aesthet Surg 2012;65:791-7.

11. Hill HL, Hester R, Nahai F. Covering large groin defects with the tensor fascia lata musculocutaneous flap. Br J Plast Surg 1979;32:12-4.

12. Rifaat MA, Abdel Gawad WS. The use of tensor fascia lata pedicled flap in reconstructing full thickness abdominal wall defects and groin defects following tumor ablation. J Egypt Natl Canc Inst 2005; 17:139-48.

13. Rozen WM, Ashton MW, Pan WR, et al. Anatomical variations in the harvest of anterolateral thigh flap perforators: a cadaveric and clinical study. Microsurgery 2009;29:16-23.

14. Kim JT, Ho SY, Hwang JH, et al. Perineal perforator-based island flaps: the next frontier in perineal reconstruction. Plast Reconstr Surg 2014;133:683e-7e.

15. Sinna R, Qassemyar Q Benhaim T, et al. Perforator flaps: a new option in perineal reconstruction. J Plast Reconstr Aesthet Surg 2010;63:e766-74.

16. Santanelli F, Berlin O, Fogdestam I. The combined tensor fasciae latae/rectus femoris musculocutaneous flap: a possibility for major soft tissue reconstruction in the groin, hip, gluteal, perineal, and lower abdominal regions. Ann Plast Surg 1993;31:168-74. 